\title{
Philosophiques
}

\section{Mixtes et passages du local au global chez Lautman : préfigurations de la théorie des faisceaux}

\section{Fernando Zalamea}

Volume 37, numéro 1, printemps 2010

Albert Lautman, philosophe des mathématiques

URI : https://id.erudit.org/iderudit/039710ar

DOI : https://doi.org/10.7202/039710ar

Aller au sommaire du numéro

Éditeur(s)

Société de philosophie du Québec

ISSN

0316-2923 (imprimé)

1492-1391 (numérique)

Découvrir la revue

Citer cet article

Zalamea, F. (2010). Mixtes et passages du local au global chez Lautman : préfigurations de la théorie des faisceaux. Philosophiques, 37(1), 17-25.

https://doi.org/10.7202/039710ar
Résumé de l'article

Nous étudions le Rapport (1935) de Lautman qui ouvre ce volume. Après une description critique des contenus et une discussion des fonds conceptuels du Rapport, nous montrons comment l'émergence postérieure de la théorie des faisceaux répond techniquement aux questionnements et aux préfigurations de Lautman. d'utilisation que vous pouvez consulter en ligne.

https://apropos.erudit.org/fr/usagers/politique-dutilisation/ 


\title{
Mixtes et passages du local au global chez Lautman: préfigurations de la théorie des faisceaux
}

\author{
FERNANDO ZALAMEA \\ Universidad Nacional de Colombia \\ www.matematicas.unal.edu.co/ fzalamea
}

\begin{abstract}
RÉSUMÉ. - Nous étudions le Rapport (1935) de Lautman qui ouvre ce volume. Après une description critique des contenus et une discussion des fonds conceptuels du Rapport, nous montrons comment l'émergence postérieure de la théorie des faisceaux répond techniquement aux questionnements et aux préfigurations de Lautman.
\end{abstract}

\begin{abstract}
We study Lautman's Rapport (1935) which opens this volume. After a critical description and a discussion of the contents of the Rapport, we show how the emergence of sheaf theory answers technically many questions raised by Lautman ten years earlier.
\end{abstract}

Nous voulons discuter dans cet article une des problématiques centrales de l'œuvre de Lautman: le va-et-vient des passages entre le local et le global en mathématiques modernes, tant dans sa finesse technique structurale que dans sa richesse méthodologique et philosophique. L'étude détaillée du local et du global se trouve largement exposée dans les deux Thèses (1937) de Lautman, mais ses grandes idées sont déjà présentes dès le Rapport sur les travaux philosophiques entrepris par M. Lautman (1935), manuscrit inédit qui apparaît dans ce numéro. En fait, on peut comprendre spécifiquement ce Rapport comme une expression in nuce des meilleures idées du jeune philosophe autour du local et du global, où il arrive jusqu'à préfigurer, dans deux passages précis, la proche émergence de la théorie des faisceaux dans l'école française.

Dans ce qui suit, nous procéderons à (i) une révision des contenus du Rapport en ce qui concerne le local et le global; (ii) une élucidation de ce que nous pourrions appeler l' «hypothèse fondamentale " qui apparait dans le Rapport, préfigurant deux idées centrales de la théorie des faisceaux; (iii) un bref aperçu de l'émergence de la théorie des faisceaux (Leray, Cartan, Serre, Grothendieck, Godement), en soulignant les mixtes et les passages lautmaniens qui sous-tendent plusieurs progrès techniques; (iv) une discussion des enjeux théoriques à la base des passages du local au global dans les travaux divers de Lautman (Rapport, Thèses, derniers articles).

\section{Le local et le global dans le Rapport (1935)}

Le Rapport sur les travaux philosophiques entrepris par M. Lautman inclut les thèmes suivants: mathématiques et physique (p. 1, pagination en référence 
au manuscrit); existence et non-contradiction à la Hilbert, théorèmes d'existence (p. 2); conditions nécessaires et suffisantes d'existence, systèmes adjoints (p. 3); passages du local au global et hypothèse fondamentale (p. 4); le tout et la partie, procédés de définition, non-prédicativité (p. 5); définitions énumératives, récurrentes, inductives par rapport à globales, axiomatiques, topologiques (p. 6); le local et le global en géométrie: Riemann, Klein, Cartan (p. 7); géométrie différentielle en grand: Cartan (p. 8); variable complexe et continuation analytique (p. 9); conditions locales aux coefficients pour la continuation analytique globale (p. 10); propriétés globales dans les extensions de corps (p. 11); solidarités globales dans la théorie de Galois, retour à la physique (p. 12). La richesse des problématiques abordées montre bien que le Rapport constitue déjà un panorama succint des Thèses que Lautman va re-penser et écrire dans les deux années suivantes (1935-1937).

Il est surprenant que tous les thèmes forts du Rapport aient à voir avec le local et le global, même lorsque Lautman entreprend une discussion sur les procédés de définition en mathématiques (pp. 5-6), où il signale que la non-prédicativité n'est pas contradictoire en soi, mais que, plutôt, elle dépend des formes de concrétude des définitions: bénigne en cas de définitions axiomatiques globales, délicate en cas de définitions récurrentes locales. La grande vision du très jeune Lautman (27 ans) est en fait stupéfiante: solidarité structurale de la physique et des mathématiques, richesse du local et du global géométrique (variétés différentielles, variétés complexes, continuation analytique), richesse du local et du global algébrique (équations, corps, théorie de Galois), le tout couronné par une audacieuse hypothèse (voir plus avant, section II) sur les rapports structurels généraux du local et du global.

Dans le Rapport, la mathématique apparaît comme une machinerie complexe qui aborde le problème de l'existence des objets à travers un réseau de passages entre le local et le global. Dans ce réseau, les transferts et obstructions donnent lieu à quelques-unes des constructions majeures des mathématiques modernes: le prolongement analytique, la théorie de Galois, les théorèmes d'existence en théorie d'équations (algébriques ou différentielles). Le recollement adéquat des données locales (coefficients, solutions particulières) permet alors une montée progressive de la connaissance mathématique, un thème encore absent dans le Rapport, mais qui sera central dans les Thèses de 1937. L'expression technique des passages entre le local et le global est l'objet primordial du Rapport, Lautman ayant toujours en vue «le problème capital de la philosophie des sciences [qui] est sans aucun doute celui des rapports de la théorie mathématique et de l'expérience physique» (p. 1). En effet, à la fin de son Rapport, Lautman signale qu'il se propose "d'appliquer également aux théories physiques les considérations qui précèdent relativement à la structure d'un tout et de ses parties » (p. 12), et il remarque que «la notion de système physique est en effet une notion 
globale que l'on définit grâce aux intégrales premières et aux invariants intégraux d'un système d'équations différentielles» (p. 12). Il est tout à fait remarquable que, en 1935 - une cinquantaine d'années en avance sur les travaux de Gromov, Connes ou Kontsevich - , un jeune philosophe ait pu entrevoir qu'une compréhension profonde de la géométrie concrète du monde physique devait passer par une connaissance primordiale des rapports mathématiques structurels abstraits entre le local et le global.

La concision et la netteté des aperçus techniques présentés par Lautman sont exceptionnelles. Il est difficile, aujourd'hui encore, de trouver un texte si court et si limpide sur une des problématiques les plus complexes des mathématiques modernes. La "nécessité de considérer à la fois le point de vue local et le point de vue global» (p. 8, nous soulignons) est un des grands atouts de la conception lautmanienne, puisque, selon le philosophe, il serait impossible de comprendre les mathématiques modernes sans envisager le permanent va-et-vient des transferts et obstructions entre le local et le global. Pendule de la connaissance mathématique, le mouvement polaire entre une logique des voisinages et une logique des structures sous-tend la mathématique qui se fait devant les yeux mêmes de Lautman. Dans ce sens, l'étude des travaux d'Élie Cartan semble avoir été déterminante: "Nous allons exposer d'abord, en nous inspirant le plus possible des travaux de M. Cartan, comment la considération simultanée du point de vue local et du point de vue global s'est imposée à la géométrie» (p. 6, nous soulignons). La connaissance nécessaire et simultanée des deux pôles est fondamentale. Il s'agit en effet d'une tendance typique de la pensée lautmanienne, où des couples puissants (Même/Autre, continu/discontinu, global/local, etc.) donnent lieu selon une dialectique complexe qui suit toute sorte de transferts et obstructions, montées et descentes, saturations et libérations - aux mixtes mathématiques effectifs.

\section{II. «L'hypothèse fondamentale» du Rapport}

Le centre conceptuel du Rapport se trouve dans le paragraphe suivant:

a) La thèse que nous allons soutenir est la suivante: Il est impossible de reconstituer globalement un tout à partir d'éléments locaux si dans les données locales, on ne tient pas compte des exigences de structure du tout, et la détermination des conditions nécessaires et suffisantes pour la résolution d'un problème n'est le plus souvent que l'introduction, dans les données locales, des garanties qui doivent assurer à la solution une existence globale (p. 4).

Et un peu plus loin:

b) Nous voulons donc montrer que la construction d'un tout par un cheminement progressif d'éléments locaux à éléments locaux n'est possible que si des considérations globales sont déjà impliquées dans les éléments locaux (p. 5).

Nous appellerons hypothèse fondamentale de Lautman la conjonction des énoncés (a) et (b), et nous essaierons de montrer comment cette hypothèse 
fondamentale est une préfiguration des idées en jeu dans le concept de faisceau mathématique, qui sera introduit et développé une dizaine d'années plus tard par l'école française, autour de Leray, Cartan, Serre, Grothendieck, Godement. Sans entrer encore dans le détail technique des faisceaux, nous pouvons remarquer d'abord que deux idées centrales sont présentes dans les énoncés (a) et (b): d'une part - dans le domaine du possible - le fait que (i) le global puisse être reconstruit comme recollement du local si et seulement si le local est capable de réfléchir la structure du global, et, d'autre part - dans le domaine de la concrétude - le fait que (ii) l'on obtienne des théorèmes d'existence global/local si et seulement si l'on décerne des conditions de réflexion précises de la structure globale dans le local. En fait, il s'agit d'une Dialectique des Idées, dans le sens même des «Idées » lautmaniennes introduites en 1938, qui prétend résoudre partiellement l'opposition entre une «notion» de globalité et la «notion » polaire associée de localité. La première Idée (i) situe la possibilité de compréhension du global comme recollement du local grâce à des structures réfléchissantes intermédiaires, et la deuxième Idée (ii) produit la concrétude d'objets globaux grâce à la détermination effective des conditions de réflexion.

L'hypothèse fondamentale de Lautman prétend donc expliquer le passage des objets mathématiques - à partir de certaines données locales, vers une existence globale - dans les cas où la structure globale de l'éventuel objet se réfléchit dans les données initiales. La possibilité d'une saturation ou d'un recollement de l'information est d'ailleurs liée à des conditions concrètes de bonne couverture ou de cohérence (condition $(b)$ : «cheminement progressif» du local dans le local). Ainsi, une sorte de "filtre lautmanien» permet d'aborder une montée idéale vers le global au cas où il faudrait tenir compte des médiations structurales liées à des recouvrementes cohérents du réel.

L'hypothèse fondamentale de Lautman combine plusieurs niveaux de pensée mathématique: les techniques propres du recollement des objets (a) «structure» globale, (b) «cheminement progressif» local, les hiérarchies modales (i) possibilité, (ii) actualités liées aux conditions concrètes de recollement, la Dialectique supérieure qui gouverne le va-et-vient local/global. Nous nous trouvons alors en face d'un mixte de second niveau, pour ainsi dire, dans la conception générale des mathématiques selon Lautman. Les "mixtes» usuels, que Lautman étudie dans sa Thèse principale, vivent, à un premier niveau, dans le spectre des «mathématiques effectives " (terme selon Lautman et Cavaillès, c.-à-d. dans le spectre des objets de la pratique quotidienne des mathématiciens). Ici, l'hypothèse fondamentale s'élève bien audelà d'une mixture mathématique concrète (même au-delà des exemples majeurs : surface de Riemann, espace de Hilbert), et agit, à un second niveau, comme indicatrice des fortes tensions d'un panorama bien plus vaste. L'émergence de la théorie des faisceaux montrera combien les idées de Lautman autour de cette Dialectique étaient justes. 


\section{L'émergence des faisceaux}

Nous évoquons brièvement dans cette section quelques percées techniques autour de la théorie des faisceaux ${ }^{1}$ entre 1942 et 1958 , en les situant par rapport aux conceptions lautmaniennes. Après les travaux de de Rham (1932) et d'Alexander (1938) sur l'idée de chaîn(e) complex(e) (le «e» distingue l'anglais et le français), et après les travaux des années trente, de Picard, Lefschetz, Steenrod, qui étudient la topologie d'une variété donnée grâce à des projections sur des variétés de dimension inférieure, et, en particulier, grâce à l'information cohérente des fibres associées aux projections, Jean Leray introduit (1946) le concept de faisceau pour contrôler les problèmes de stabilité et de variation dans les fibres. Les travaux de Leray proviennent de ses recherches sur les équations différentielles (index et couvertures), réalisées au camp d'officiers Oflag XviI, et se développent dans ses cours au Collège de France (1947-1950), en liaison avec les suites spectrales et une opératoire générale (images, quotients, limites) des nouveaux objets. Dès l'apparition des faisceaux, ceux-ci entrent donc naturellement en rapport avec quelques conceptions centrales chères à Lautman: la problématique platonicienne-lautmanienne de l'Un et du Multiple (stabilité, variation), le dynamisme propre des mathématiques effectives (projections, recollements), le va-et-vient des montées et descentes entre le particulier (équations différentielles, variétés) et le général (opératoire abstraite des faisceaux).

La théorie des faisceaux va se développer dans le séminaire de Henri Cartan à l'École normale supérieure, et va acquérir sa forme définitive déjà autour des années 1948-1951. Lazard et Cartan donnent une présentation «topologique $»^{2}$ où les fibres sont des modules bien agencés sur un espace topologique sous-jacent; la structure des «modes de définition» des faisceaux est inmédiatement mise en lumière, comparant les présentations selon homéomorphismes locaux et selon modules de sections ${ }^{3}$. La vision structurale de Lautman, fortement remarquée par Dieudonné dans son introduction à l'édition 10/18 des œuvres ${ }^{4}$, est bien sûr très vive dans le séminaire Cartan, dans les mêmes années que celles de l'éclosion de Bourbaki. Mais il est fort intéressant que l'expression technique de Cartan arrive à réfléchir une forme aussi fidèle que l'expression conceptuelle de l' «hypothèse

1. Pour des bonnes introductions historiques et conceptuelles à la théorie des faisceaux, voir John Gray, "Fragments of the history of sheaf theory ", Lecture Notes in Mathematics 753, Berlin, Springer, 1979, pp. 1-79, ou Christian Houzel, "Histoire de la théorie des faisceaux ", La géométrie algébrique, Paris, Albert Blanchard, 2002, pp. 293-304.

2. Henri Cartan, Séminaire $3^{\mathrm{e}}$ année 1950-1951 - Cohomologie des groupes, suite spectrale, faisceaux, Paris, École normale supérieure, 1955 (exposé de H. Cartan, «Faisceaux sur un espace topologique I", le 9-4-1951, page 14-01).

3. Ibidem, pages 14-02, 14-03.

4. Introduction (1977) reprise dans Albert Lautman, Les mathématiques, les Idées et le réel physique, Paris, Vrin, 2006, pp. 35-38. 
fondamentale»du Rapport de Lautman: les «exigences de structure du tout»(Lautman) correspondent aux agencements structurels des modules de sections (Cartan), et les «garanties» et «cheminements progressifs » dans le local (Lautman) correspondent aux conditions d'homéomorphisme local (Cartan). Il est également remarquable que les travaux de Cartan sur l'homologie d'espaces compacts de fonctions holomorphes (1945) et sur les idéaux de fonctions holomorphes (1950) - formes modernes de l'héritage classique (Gudermann, Weierstrass) autour du concept de continuation analytique - se trouvent liés à des considérations sur les faisceaux de modules complexes, en plein accord avec l'urgence des problèmes de rattachement entre le local et le global du point de vue des fonctions analytiques, soulignés dans le Rapport.

Les faisceaux algébriques cohérents (FAC) de Serre (finement engendrés par sections, 1955-1956) aident à préciser une très forte propriété de préservation de la vérité: pour les $\mathrm{FAC}$, les théorèmes vrais dans un point sont vrais aussi sur tout un voisinage du point. Il s'agit ici d'une propriété capitale de continuité de la logique des faisceaux («sheaf logic») — développée par Joyal et, sous une forme plus souple, par Caicedo ${ }^{5}$ - qui correspond à un des paris conceptuels majeurs de l'œuvre de Lautman. En fait, à un moment difficile, le jeune normalien s'est érigé contre une "démission ${ }^{6}$ " de la philosophie aux dépens du langage, a souhaité «à la philosophie des sciences une ambition plus haute ${ }^{7}$ », et a imaginé un programme de compréhension des mathématiques modernes - non analytique, discret ou statique - ouvert vers une synthèse continue et dynamique du savoir (ce qui explique son penchant profond pour un platonisme non réifié, ouvert au mouvement, suivant Natorp et Robin). Il est étonnant que la logique des faisceaux montre - en accord avec le philosophe - que cette connaissance continue de la vérité est possible. Ainsi, en lisant Lautman, on arrive à "écouter la voix des choses» (Grothendieck) et à percevoir quelques préfigurations conceptuelles qui, plus tard, vont se traduire techniquement dans les mathématiques effectives.

Selon une Dialectique générale qui tient compte d'une authentique oscillation pendulaire, une analyse/synthèse du concept de faisceau peut se réaliser suivant quelques lignes lautmaniennes ${ }^{8}$. Partant du couple de notions polaires Même/Autre, une des problématiques essentielles de la phénoménologie consiste à étudier le réseau de passages entre ces pôles. Comme instances mathématiques du couple Même/Autre, on trouve d'autres couples

5. Xavier Caicedo, "Lógica de los haces de estructuras", Revista de la Academia Colombiana de Ciencias Exactas, Físicas y Naturales XIX (74) (1995), pp. 569-585.

6. Lautman, Les mathématiques..., p. 50 (1933).

7. Ibidem, p. 64 (1936).

8. Les catégories utilisées (notions/Idées; mixtes; Autre/Même; local/global) sont fondamentales chez Lautman. Notre usage de ces termes pour clarifier le concept de faisceau n'apparaît pas, bien sûr, dans son œuvre, mais l'interprétation s'accorde pleinement avec son esprit. 
de notions: intégration/différentiation, global/local. L'étude des passages et des mixtes entre le local et le global — raison d'être de la théorie des faisceaux - devient alors une problématique centrale des mathématiques contemporaines (Grothendieck, Atiyah, Connes, Gromov, etc.), Une analyse mathématique des notions de localité donne lieu à des techniques de couverture cohérente du local, tandis qu'une synthèse mathématique donne lieu à des objets globaux obtenus par recollement adéquat des couvertures. Des mixtes mathématiques précis se manifestent donc, tant d'un point de vue analytique - voisinage, couverture (recouvrement), cohérence, recollement - que d'un point de vue synthétique - sections, préservations, projections, restrictions. Les concepts de préfaisceau (Grothendieck) et de faisceau (Leray, Cartan) permettent d'organiser alors les passages entre le local et le global, selon le diagramme suivant d'Idées (résolutions partielles de paires de notions):

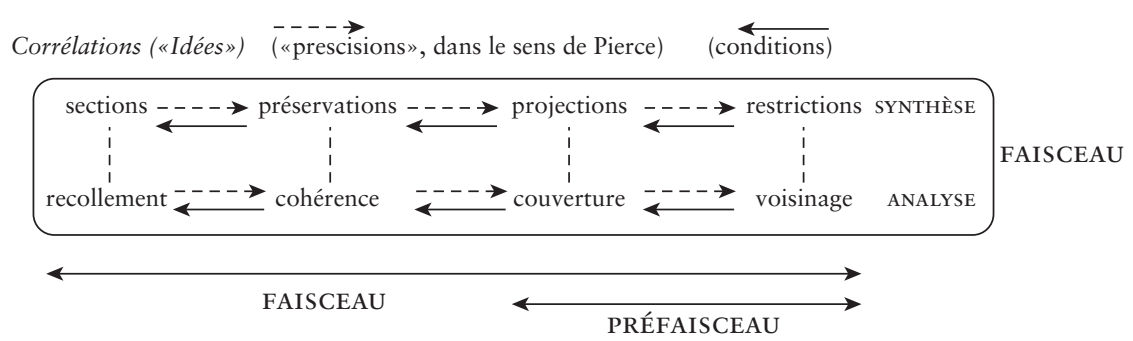

\section{Obstructions et transferts du local au global}

Dans cette section finale nous mettons en rapport l'«hypothèse fondamentale» de Lautman avec notre «analyse/synthèse » du concept de faisceau, et nous signalons comment les passages mathématiques local/global soustendus par ce rapport forment une sorte de colonne vertébrale de l'œuvre du philosophe. L' «hypothèse fondamentale» du Rapport se divise en deux affirmations centrales: (1) pour qu'il existe une possibilité de passage du local au global, il faut que la structure du global soit réfléchie dans le local; (2) l'actualité $d u$ passage concrète s'obtient lorsqu'on détermine précisément les conditions structurales de réflexion. Il s'agit de deux instances corrélatives, qui «vivent» dans la priméité et dans la secondéité selon Peirce (espaces du possible et de l'actuel), et qui correspondent aux deux temps fondamentaux de l'opératoire des faisceaux: (1) possibilité de recollement par le biais des préservations et des sections; (2) acte de recouvrement par le biais des restrictions et des projections (voir notre figure: analyse/synthèse du concept de faisceau).

La problématique générale des passages entre local et global, adressée dans le Rapport et raison d'être de la théorie des faisceaux, correspond 
alors, selon une perspective lautmanienne, à l'étude d'un réseau complexe d'obstructions et de transferts représenté dans la figure suivante:

\begin{tabular}{|c|c|c|c|c|}
\hline $\begin{array}{c}\text { Ruptures } \\
\text { de continuité }\end{array}$ & \multicolumn{3}{|c|}{ Éléments idéaux / saturations } & Complétude \\
\hline Réel initial & \multicolumn{3}{|c|}{ Mixtes / Idées } & Réel épanoui \\
\hline \multirow{2}{*}{$\begin{array}{l}\text { Fragments } \\
\text { locaux }\end{array}$} & (1) & Faisceaux & $(2)$ & \multirow{2}{*}{$\begin{array}{l}\text { Existence } \\
\text { globale }\end{array}$} \\
\hline & $\mathrm{Ob}$ & & & \\
\hline
\end{tabular}

Les mathématiques se développent tout au long de fortes tensions entre le Réel et l'Idéal (se rappeler de la présence de la physique au début et à la fin du Rapport), grâce à une très riche Dialectique intermédiaire où émergent les grandes constructions techniques (groupe de Galois, surface de Riemann, espace de Hilbert, etc.) À partir d'un Réel initial, parsemé de creux d'information et de ruptures de continuité, les mathématiques contribuent à saturer les concepts hiérarchiques en jeu, en introduisant des éléments idéaux complexes9. En fait, la construction de ces objets idéaux s'accomplit grâce à des mixtes répondant à des obstructions bien définies, qui sont ensuite résolues - souvent par saturation - et donnent lieu enfin à de bons transferts d'information ${ }^{10}$. C'est en particulier le cas des faisceaux, qui permettent de passer du local au global si jamais ils dépassent quelques obstructions (mauvais recouvrements, incohérences locales). Si ce dépassement est possible, c'est-à-dire si la structure du global se voit réfléchie dans le local, les transferts d'information mathématique se réalisent en saturant le faisceau, c'est-à-dire en déterminant de façon complète les conditions locales de réflexion de la structure globale.

L'examen vigilant des passages du local au global a été permanent dans l'œuvre de Lautman. Dès ses Considérations sur la logique mathématique

9. Cette lecture lautmanienne correspond à la vision de Hilbert dans «Sur l'infini» (1925).

10. Quelques exemples modernes considérés par Lautman: $a$ ) Galois, étude des racines algébriques (locales) par le biais de l'action (globale) du groupe de Galois sur des corps médiateurs; obstruction: simplicité du sous-groupe An pour $\mathrm{n} \geq 5$; transfert: résolution par radicaux si le groupe est soluble; $b$ ) Riemann : étude des solutions différentielles (locales) par le biais des objets géométriques et invariants topologiques (globaux); obstruction: non-préservation de connexité, compacité, etc.; transfert: théorèmes qualitatifs d'existence et de représentation (conforme, etc.); c) Hilbert: existence (globale) par le biais de la consistance déductive (locale); obstruction: relativité de la consistance à partir du seuil de l'arithmétique de Peano (incomplétude de Gödel); transfert: preuves d'équiconsistance et de consistance relative. Exemple contemporain majeur, encore non disponible pour Lautman; $d$ ) Grothendieck: dialectique forme/structure (globale) par le biais des schémas, topos, motifs (locaux à un deuxième niveau, vus à partir des perspectives relatives grothendickiennes: variétés variables, ensembles variables, foncteurs variables); obstruction: cohérences fonctorielles; transfert: cohomologies, «les plus puissants outils du siècle» selon Grothendieck. 
(1933), où les champs de Herbrand sont envisagés du point de vue complémentaire de la consistance locale et de l'existence globale, jusqu'à son dernier travail, Le problème du temps (1943), où il considère la topologie globale de l'Univers en rapport avec l'orientation locale du temps, Lautman a révisé toute sorte de situations autour d'une Dialectique générale du local et du global. Comme nous l'avons vu, le Rapport (1935) - apparemment son deuxième écrit - est dédié en entier à cette grande Dialectique. Ses deux Thèses (1937) apportent un très grand nombre d'exemples et de précisions additionnelles ${ }^{11}$. Finalement, à la mémorable session de la Société française de philosophie (1939), Lautman signale comment l'abstraction moderne est nécessairement liée à des considérations de globalité et de structure.

Étant donné l'ampleur et la justesse visionnaire des considérations lautmaniennes autour de la Dialectique local/global, et étant donné le rôle majeur que la théorie des faisceaux a exercé dans la mathématique contemporaine (à partir de Cartan, Serre, Grothendieck), nous sommes forcés d'observer encore une fois l'extraordinaire pertinence des travaux de Lautman pour la philosophie, non seulement moderne, mais contemporaine des mathématiques. Au centenaire de sa naissance, son héritage semble être plus vif que jamais.

11. Quelques thèmes étudiés dans la Thèse principale: fonctions analytiques, Weierstrass (local) par rapport à Riemann (global); géométrie riemannienne, coordonnées locales par rapport à des extensions globales, métriques locales par rapport à des propriétés globales; cosmologie: structure globale de l'Univers par rapport aux lois locales (Cartan); espaces de fonctions continues: représentations (locales) en série par rapport à la complétude (globale) de l'espace; corps de classes: ramification locale des idéaux par rapport aux propriétés globales des extensions; surfaces de Riemann: uniformisations locales par rapport aux uniformisations globales; équations différentielles: solutions locales par rapport à l'existence qualitative globale (Poincaré); fonctions modulaires (systèmes locaux) par rapport aux fonctions automorphes (uniformisantes globales). Quelques thèmes étudiés dans la Thèse complémentaire: espaces vectoriels, structure globale par rapport aux bases locales; groupes: actions (globales) par rapport aux nombres (locaux); anneaux: décompositions du tout (anneau) par rapport à la partie (centre). 